\title{
Repeat physical stress echocardiography in asymptomatic severe aortic stenosis
}

\author{
Elena Ferrer-Sistach, Josep Lupón, Silvia Serrano-García, Albert Teis-Soley, \\ Francisco Gual-Capllonch, Gladys Juncà-Puig, Nuria Vallejo-Camazón, \\ Jorge López-Ayerbe, Antoni Bayés-Genís \\ Heart Institute, Hospital Universitari Germans Trias i Pujol, Badalona, Spain; \\ Department of Medicine, Autonomous University of Barcelona, Barcelona, Spain; \\ and CIBERCV, Instituto Carlos III, Madrid, Spain
}

In severe aortic stenosis (SAS), the presence of symptoms is associated with ominous prognosis with 5 -year mortality of $15-50 \%$ [1], and is a wellestablished indication for valve replacement [2]. On the other hand, in asymptomatic SAS, case management requires clinical skills, and the timing of valve replacement is unclear. Holistic assessment of the clinical situation (including co-morbidities and frailty), risk stratification, and complementary tests are critical for managing asymptomatic SAS and determining optimal valve replacement timing.

In clinical practice, at the time of SAS diagnosis, approximately $50 \%$ of patients report being asymptomatic in their day-to-day life [1]. However, this may be due to an unconscious adaptive process causing them to limit the intensity of their physical activity. Thus, it can be difficult to determine whether a patient truly lacks symptoms, especially in elderly patients. Stress testing is useful for exposing symptoms, and is a safe technique for use in stable patients [3]; clinical practice guidelines recommend the use of stress testing when assessing asymptomatic SAS patients [2]. Valve replacement is indicated in patients who exhibit clinical signs during stress testing, or lower blood pressure during physical activity. However, it remains unclear whether it is of value to repeat a stress test to uncover symptoms in asymptomatic SAS.

The incidence of a positive stress test in SAS ranges from $15 \%$ to $65 \%$ [3]. Despite this variability, stress testing exhibits good negative predictive value for cardiovascular events [4]. Performing echocardiographic assessment before and after the stress test (physical stress echocardiography [PE]) provides additional physiological parameters that can help establish the cause of symptoms [5].

From June 2014 to August 2019, the value of $\mathrm{PE}$ and repeat PE during follow-up in 85 asymptomatic SAS patients who were prospectively enrolled in a specialized valve clinic (Table 1) was investigated. Baseline PE was the key indicator for aortic valve replacement in $23(27 \%)$ patients: $8(9.4 \%)$ had dyspnea, $2(2.4 \%)$ angina, and $16(18.8 \%)$ abnormal blood pressure response. Furthermore, 17 (20\%) exhibited electric changes suggestive of ischemia, $\mathrm{PE}$ revealed a median gradient increase of $>20 \mathrm{mmHg}$ during stress in $31(36.9 \%)$ patients, ventricular dysfunction in $2(2.4 \%)$ patients, and segmental disorders in $8(9.5 \%)$ patients. Of the alterations observed during $\mathrm{PE}$, those which were the reason to indicate valve replacement were only symptoms and abnormal blood pressure response. The rest of the information obtained helped to make the decision but it was not the main reason.

A second $\mathrm{PE}$ was performed in 27 patients (median time 16 months after the first PE), and a third $\mathrm{PE}$ was completed in 5 patients (median time 16 months after the second PE). The second PE was the key indication for aortic valve replacement in $9(33.3 \%)$ patients; it was clinically positive by symptoms in $6(22.2 \%)$ patients: $3(12 \%)$ patients had dyspnea, and $4(14.8 \%)$ angina and abnormal blood pressure response was found in $4(14.8 \%)$ patients. Electric changes occurred in $9(33.3 \%)$ patients, and a median gradient increase of $>20 \mathrm{mmHg}$

Address for correspondence: Antoni Bayes-Genis, MD, PhD, FESC, Head, Heart Institute. Hospital Universitari Germans Trias i Pujol, Carretera de Canyet s/n 08916, Badalona (Barcelona), Spain, e-mail: abayesgenis@gmail.com 
Table 1. Data of asymptomatic severe aortic stenosis patients undergoing physical stress echocardiography.

\begin{tabular}{lc}
\hline Demographic data & $32(37.6)$ \\
Women & $74.1 \pm 9.4$ \\
Age [years] & $9(10.6)$ \\
Smokers & $70(82.3)$ \\
High blood pressure & $30(35.3)$ \\
Diabetes mellitus & $65(76.4)$ \\
Dyslipidemia & \\
Echocardiographic data & $22(25.9)$ \\
Bicuspid aortic valve & $4.3 \pm 0.3$ \\
Peak aortic velocity [m/s] & $74.5 \pm 11.1$ \\
Maximum aortic gradient [mmHg] & $47 \pm 8$ \\
Mean aortic gradient [mmHg] & $0.77 \pm 0.12$ \\
Aortic valve area [cm $\left.{ }^{2}\right]$ & $67.9 \pm 0.12$ \\
LVEF [\%] & \\
Blood count & $294.0(148-661)$ \\
NT-proBNP [ng/L] & \\
\hline
\end{tabular}

Data presented as number (\%), mean \pm standard deviation, or median and percentiles of 25-75 (Q1-Q3). LVEF — left ventricular ejection fraction; NT-proBNP - N-terminal pro-B-type natriuretic peptide

in $15(55.5 \%)$ patients. Finally the third PE was the key indicator for aortic valve replacement in $2(40 \%)$ patients, because it was clinically positive by symptoms.

Present findings indicated that repeating $\mathrm{PE}$ during follow-up was useful for asymptomatic SAS management. Indeed, the key indication for valve replacement increased from $27 \%$ of cases at baseline $\mathrm{PE}$ to $33 \%$ in the second $\mathrm{PE}$, and $40 \%$ in the third PE. This pilot study in patients with asymptomatic SAS demonstrated that $\mathrm{PE}$ at both the first visit and during follow-up was useful in indicating valve replacement. Further studies in larger cohorts are needed to confirm these findings, and to establish the optimal time-frames for serial $\mathrm{PE}$ in such patients.

\section{Funding}

Elena Ferrer-Sistach was supported by grants from Societat Catalana de Cardiologia.

Conflict of interest: None declared

\section{References}

1. Pellikka PA, Sarano ME, Nishimura RA, et al. Outcome of 622 adults with asymptomatic, hemodynamically significant aortic stenosis during prolonged follow-up. Circulation. 2005; 111(24): 3290-3295, doi: 10.1161/CIRCULATIONAHA.104.495903, indexed in Pubmed: 15956131.

2. Baumgartner H, Falk V, Bax JJ, et al. 2017 ESC/EACTS Guidelines for the management of valvular heart disease. Eur Heart J. 2017; 38(36): 2739-2791, doi: 10.1093/eurheartj/ehx391, indexed in Pubmed: 28886619.

3. Redfors B, Pibarot P, Gillam LD, et al. Stress testing in asymptomatic aortic stenosis. Circulation. 2017; 135(20): 1956-1976, doi: 10.1161/CIRCULATIONAHA.116.025457, indexed in Pubmed: 28507251.

4. Rafique AM, Biner S, Ray I, et al. Meta-analysis of prognostic value of stress testing in patients with asymptomatic severe aortic stenosis. Am J Cardiol. 2009; 104(7): 972-977, doi: 10.1016/j. amjcard.2009.05.044, indexed in Pubmed: 19766766.

5. Ennezat PV, Maréchaux S, Iung B, et al. Exercise testing and exercise stress echocardiography in asymptomatic aortic valve stenosis. Heart. 2009; 95(11): 877-884, doi: 10.1136/ hrt.2008.150011, indexed in Pubmed: 18812409. 